\title{
Measuring a Quantum Dot with an Impedance-Matching On-Chip Superconducting $L C$ Resonator at Gigahertz Frequencies
}

\author{
M.-C. Harabula, ${ }^{1}$ T. Hasler, ${ }^{1}$ G. Fülöp, ${ }^{1}$ M. Jung, ${ }^{1,2}$ V. Ranjan, ${ }^{1,3}$ and C. Schönenberger ${ }^{1}$ \\ ${ }^{1}$ Department of Physics, University of Basel, Klingelbergstrasse 82, CH-4056 Basel, Switzerland \\ ${ }^{2}$ DGIST Research Institute, DGIST, 333 TechnoJungang, Hyeongpung, Daegu 42988, Korea \\ ${ }^{3}$ Quantronics Group, SPEC, CEA, CNRS, Université Paris-Saclay,
}

CEA Saclay, F-91191 Gif-sur-Yvette, France

(Received 31 July 2017; published 6 November 2017)

\begin{abstract}
We report on the realization of a bonded-bridge on-chip superconducting coil and its use in impedance matching a highly ohmic quantum dot (QD) to a 3-GHz measurement setup. The coil, modeled as a lumped-element $L C$ resonator, is more compact and has a wider bandwidth than resonators based on coplanar transmission lines (e.g., $\lambda / 4$ impedance transformers and stub tuners), at potentially better signalto-noise ratios. Specifically, for measurements of radiation emitted by the device, such as shot noise, the $50 \times$-larger bandwidth reduces the time to acquire the spectral density. The resonance frequency, close to $3.25 \mathrm{GHz}$, is 3 times higher than that of the one previously reported, a wire-bonded coil. As a proof of principle, we fabricate an $L C$ circuit that achieves impedance matching to an approximately $15-\mathrm{k} \Omega$ load and validate it with a load defined by a carbon nanotube QD, whose shot noise we measure in the Coulombblockade regime.
\end{abstract}

DOI: 10.1103/PhysRevApplied.8.054006

\section{INTRODUCTION}

Superconducting qubit control and readout of very resistive quantum devices need microwave (MW) resonators operating at frequencies in the range of $1-10 \mathrm{GHz}$. To efficiently measure radiation emitted from a device, one attempts to couple the high device impedance to the $Z_{0}=$ $50 \Omega$ characteristic impedance of the usual coaxial lines using an impedance-matching circuit. These circuits are often implemented with superconducting on-chip transmission lines. Examples include the quarter-wavelength step transformer in the fluxonium qubit [1], stub tuners for quantum point contacts [2], and quantum dots (QDs) $[3,4]$. An alternative approach makes use of an $L C$ resonator built either from a lumped-element inductor [5] or form on-chip coils [6]. Nonetheless, the resonance frequencies have typically been limited to a maximum of about $1 \mathrm{GHz}$ [7-9]. It is, however, important to improve $L C$ circuits since they are much less demanding in chip footprint than transmission line circuits are. While a transmission line circuit at $5 \mathrm{GHz}$ needs up to about $1 \mathrm{~cm}$ in length, meandered on 1-3 $\mathrm{mm}^{2}$, an appropriate on-chip coil can be fabricated on a $10 \times$ to $30 \times$ smaller area. This is an important consideration for the future scaling of qubit networks for quantum processors. To understand the challenge, the key parameters of an $L C$ matching circuit are its resonance frequency, given by $1 /(2 \pi \sqrt{L C})$, and its characteristic impedance $Z_{c}$, defined as $\sqrt{L / C}$. In order to match to a high-impedance load, $Z_{c}$ should be as large as possible, meaning that one has to aim for a large inductance. At the same time, the resonance frequency should stay high, constraining the capacitance to the smallest possible values. In fact, the limiting factor in achievable resonance frequency is the residual stray capacitance in the circuit, which we minimize in our approach.

Addressing the compactness of MW resonators in this work, we fabricate a $200-\mu \mathrm{m}$-wide on-chip superconducting coil with a wire-bonded bridge (Fig. 1) and utilize it as a lumped $L C$ matching circuit in a carbon-nanotube (CNT) QD noise experiment at a working frequency of close to $3.25 \mathrm{GHz}$. Compared to the one previously reported case of an on-chip inductor with a bonded bridge $[8,10]$, we achieve a threefold frequency increase and a similar footprint decrease. Comparable compactness has been achieved only with Josephson-junction arrays acting as quarterwavelength resonators $[11,12]$.

\section{SAMPLE FABRICATION}

A planar spiral inductor raises the issue of connecting its inner end to the rest of the circuit. While it is possible to fabricate this bridge by lithography, we realize that the close proximity of the bridge to the coil adds substantial capacitance, limiting the performance of the $L C$ circuit. In addition, the microfabrication of air bridges of this kind add several fabrication steps. That is why we develop a solution of bonding a wire between a geometry-restricted inner pad and an external bigger pad at the low-impedance end.

The fabrication starts with the preparation of a region on the undoped $\mathrm{Si} / \mathrm{SiO}_{2}(500-\mu \mathrm{m} / 170-\mathrm{nm})$ substrate where CNTs will be placed. The $\mathrm{Si}$ crystal parameters are orientation $\langle 100\rangle, \rho>100 \Omega \mathrm{m}$. Thus, we first evaporate 

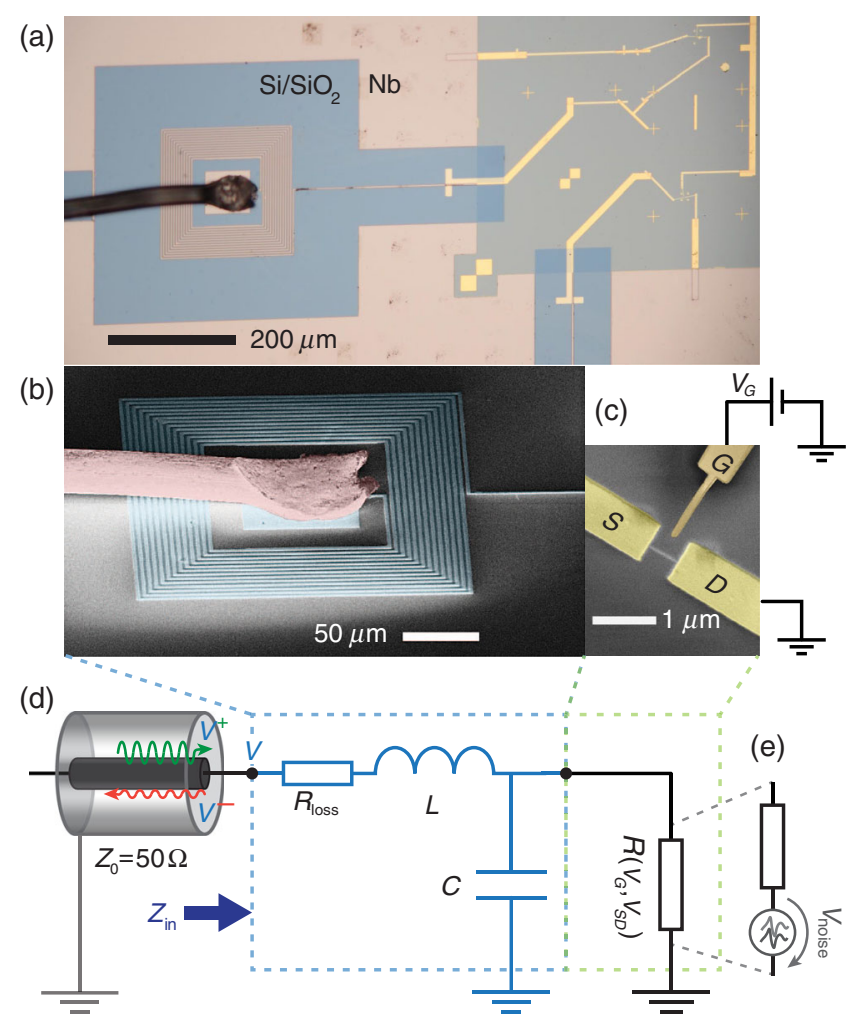

FIG. 1. (a) Optical micrograph of the sample. (b) False-color SEM image of a superconducting $\mathrm{Nb}$ on-chip coil (cyan) connected via an $\mathrm{Al}$ bond wire (pink) to the measurement setup. The right end of the coil connects to a QD laying on the same $\mathrm{Si} / \mathrm{SiO}_{2}$ substrate. (c) False-color SEM image of the CNT QD with $\mathrm{Ti} / \mathrm{Au}$ leads $(S, D)$ and a side gate $(G)$. (d) Simplified sketch of the microwave-only electrical circuit: the coil is modeled as an $L C$ circuit whose losses are caught by a resistance $R_{\text {loss }}$ in series with the inductor $L$, the CNT is characterized by a resistance dependent on the gate and source-drain dc voltages, and the $L C$ tank circuit partially matches $R$ to the $Z_{0}=50 \Omega$ of the measurement setup line. In reflectance measurement, the voltage $V=V^{+}+V^{-}$at the interface is the sum of the voltages given by the incident wave (green) and the reflected wave (red). (e) In noise measurements, there is no incident wave, $V^{+}=0$. By contrast, a voltage source $V_{\text {noise }}$ needs to be considered in series with the CNT resistance. This voltage is transmitted to become $V$ with a frequency-dependent transmission function determined by the $L C$ network.

$\mathrm{Ti} / \mathrm{Au}(10 \mathrm{~nm} / 30 \mathrm{~nm})$ for markers and CNT partial contacts in a square area [the top-right corner in Fig. 1(a)]. We then protect it with a PMMA/hydrogen-silsesquioxane bilayer resist. Afterwards, we sputter $100 \mathrm{~nm}$ of $\mathrm{Nb}$ and lift the protection bilayer resist off. Subsequently, we $e$-beam pattern bonding pads and the desired inductor in a new PMMA resist layer, then etch the $\mathrm{Nb}$ film with an $\mathrm{Ar}^{-\mathrm{Cl}_{2}}$ inductively coupled plasma; the surrounding $\mathrm{Nb}$ becomes the ground plane. Next, we stamp CNTs $[4,13]$ in the predefined region, locate them using a scanning electron microscope (SEM), and contact the chosen CNT in one $\mathrm{Ti} / \mathrm{Au}$ evaporation step to the coil and to ground
[Fig. 1(a)], utilizing the partial contacts. In the same lithography step, a side gate is created at a distance of approximately $300 \mathrm{~nm}$ from the CNT [Fig. 1(c)]. With the device glued onto a sample holder, we use $\mathrm{Al}$ wire to bond the remaining end of the coil to a neighboring bigger pad. Because of the relatively small size of the inner pad $(70 \times 70 \mu \mathrm{m}$, barely larger than the bonder wedge), the man-operated bonding is delicate [Fig. 1(b)]. Finally, we connect the latter pad to the MW line of the printed-circuitboard sample holder. Furthermore, the $\mathrm{Nb}$ ground plane of the sample is bonded with multiple wires along the wafer edge to the sample-holder ground plane.

The square spiral inductor used in this experiment [Figs. 1(a) and 1(b)] has an outer dimension of $210 \mu \mathrm{m}$, 14 turns with width $w=2 \mu \mathrm{m}$ and spacing $s=2 \mu \mathrm{m}$. In the device investigated here, two of the turns are shorted, lowering the effective inductance and thus shifting up the resonance frequency by several percent.

\section{HIGH-FREQUENCY SETUP AND RESONATOR CHARACTERIZATION}

The simplified schematic of the MW circuit is illustrated in Fig. 1(d). The impedance-matching circuit, contained in the light-blue dashed rectangle, is modeled with lumped elements: $L$ is the inductance of the coil, $C$ its capacitance to ground, and $R_{\text {loss }}$ the effective loss resistance, accounting for the rf loss of the superconducting material and the dielectric loss in the substrate. The input impedance $Z_{\text {in }}$ [Fig. 1(d)] reads

$$
Z_{\text {in }}=R_{\mathrm{loss}}+i \omega L+\frac{1}{G+i \omega C}
$$

with $G=1 / R$ being the load differential conductance. $Z_{\text {in }}$ can be approximated at $\omega_{0}=1 / \sqrt{L C}$ by

$$
Z_{\text {in }}\left(\omega_{0}\right) \approx R_{\text {loss }}+\frac{Z_{c}^{2}}{R}
$$

This approximation is valid for $\left|Z_{c}\right| \ll R$. Full matching is achieved at $\omega_{0}$ when $Z_{\text {in }}\left(\omega_{0}\right)=Z_{0}$. Thus, one obtains the condition $Z_{c}=\sqrt{\left(Z_{0}-R_{\text {loss }}\right) R}$, meaning that the characteristic impedance of the $L C$ circuit should be equal to the geometric mean of $Z_{0}$ and $R$ if we neglect $R_{\text {loss }}$. Typical values for $Z_{c}$ are between 1 and a few $\mathrm{k} \Omega$.

In reflectance measurements, a continuous sinusoidal wave is applied to the $L C$ circuit, where it appears with amplitude $V^{+}$. Part of the incident signal is reflected back with amplitude $V^{-}$due to impedance mismatch. The reflection coefficient $\Gamma$ is given by

$$
\Gamma \equiv \frac{V^{-}}{V^{+}}=\frac{Z_{\text {in }}-Z_{0}}{Z_{\text {in }}+Z_{0}} .
$$


(a)

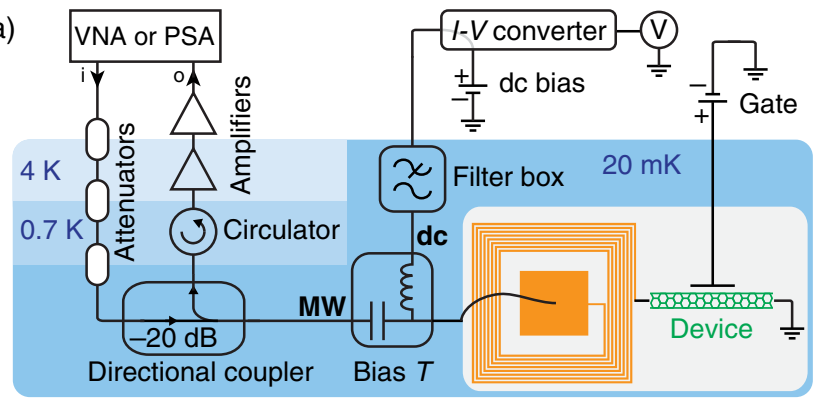

(b)

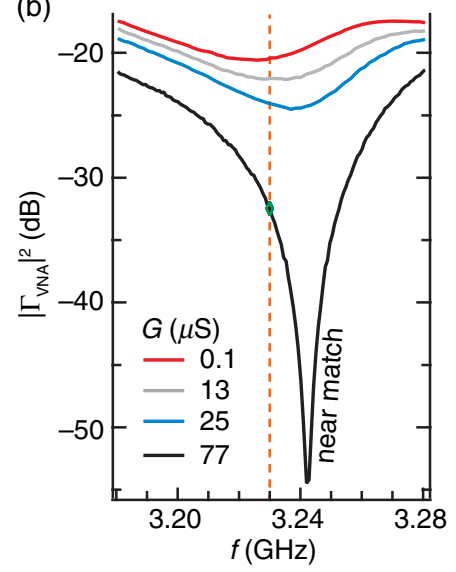

(c)

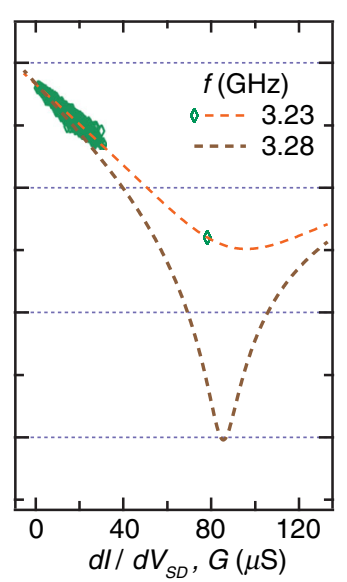

FIG. 2. (a) Schematic of the measurement setup with directcurrent (dc) input and microwave (MW) input (i) and output (o) lines. The setup is used, alternatively, for reflectometry (both MW input and output) and noise measurements (only MW output), employing either a VNA or an PSA (vector network or power spectrum analyzer). (b) Magnitude squared of the reflection coefficient $\Gamma_{\mathrm{VNA}} \equiv V_{o} / V_{i}$ measured around the resonance frequency. The further-investigated regime lies between the close-to-zero conductance (Coulomb blockade) red curve (shallowest trace) and the vicinity of the blue curve. The separately measured black trace corresponds to an almost perfectly matched conductance. (c) Fit curve (orange) of the reflectance data $\left|\Gamma_{\mathrm{VNA}}\right|^{2}\left(d I / d V_{S D}\right)$ collected at the fixed frequency $3.23 \mathrm{GHz}$ during a $V_{G} \times V_{S D} 2 \mathrm{D}$ sweep. All of the measured $d I / d V_{S D}$ values (the green diamonds) correspond to less than $30 \mu \mathrm{S}$, except for one point taken from the near-match curve in (b). The deeper curve (brown) is calculated with the extracted fit parameters at the extracted resonance frequency, and its minimum indicates $G_{\text {match }}$.

The magnitude squared of the reflection coefficient is also referred to as the reflectance.

The complete measurement setup, depicted in Fig. 2(a), is the same as in Ref. [4]. The load of the matching circuit, the CNT device, is tuned by $V_{S D}$ and $V_{G}$-the dc sourcedrain and gate voltages, respectively. MW lines are used either in reflectometry mode (a microwave signal is sent to the sample and a reflected signal is read from it) or in noise mode, where the MW output line conveys the noise signal produced by the sample. The two respective modes utilize a vector network analyzer (VNA) and a power spectrum analyzer (PSA).

We characterize the impedance-matching $L C$ resonator by analyzing reflectance measurements together with the simultaneously measured dc current $I$, from which we derive the differential conductance $d I / d V_{S D}$. The $L C$ parameters we thus obtain allow us to extract the QD noise from the measured noise power, as we will show.

In practice, the measured reflection coefficient is measured not for the sample but for the VNA, yielding $\Gamma_{\mathrm{VNA}} \equiv V_{o} / V_{i}$, where $o$ and $i$ refer to the VNA ports [see Fig. 2(a)]. $\Gamma_{\mathrm{VNA}}$ differs from $\Gamma$ by a frequencydependent baseline $b(f)$ produced by attenuations and amplifications in the setup

$$
\left|\Gamma_{\mathrm{VNA}}\right|^{2}=b(f) \cdot|\Gamma|^{2} .
$$

If spurious reflections occur in components across the output line, then standing waves reside in sections of it and the baseline $b(f)$ can exhibit a complicated pattern.

In principle, we need to extract five parameters, $L, C, b$, $R_{\text {loss }}$, and $R$. There are two possible approaches: one can fit the frequency dependence of $\Gamma_{\mathrm{VNA}}$ for a fixed known value of $G$-for example, for $G=0$, which is realized when the QD is deep in Coulomb blockade, where the current is almost completely suppressed. Figure 2(b) shows measured curves for three different $G$ values. Unfortunately, the baseline function $b(f)$ is also markedly frequency dependent, shifting the minimum of the curves to different values. Because of this dependence, it is already difficult to extract the resonance frequency accurately.

We therefore proceed with the second approach. We fix the frequency to a value close to resonance, in the following, to $f_{m}=3.23 \mathrm{GHz}$. We carry out simultaneous measurements of reflectance and dc current over several Coulomb diamonds. We obtain the reflectance $\left|\Gamma_{\mathrm{VNA}}\right|^{2}\left(V_{G}, V_{S D}\right)$ and the low-frequency $d I / d V_{S D}\left(V_{G}, V_{S D}\right)$ maps. For each pair $\left(V_{G}, V_{S D}\right)$, the two parallel measurements define one point in the $\Gamma_{\mathrm{VNA}}$-versus- $d I / d V_{S D}$ scatter plot. To obtain the circuit parameters, we fit to this scatter plot the theoretical dependence $\Gamma_{\mathrm{VNA}}(G)$, thereby implicitly assuming that the dc differential conductance equals the high-frequency one $\left(d I / d V_{S D}=G\right)$. We showed in previous work that the real part of the CNT admittance at similar GHz frequencies is the same as for $d I / d V_{S D}[3,4]$. The curve $\Gamma_{\mathrm{VNA}}(G)$ can be calculated using Eqs. (1), (3), and (4). It is displayed in Fig. 2(c) for a wide range of $G$ values. At the resonance frequency when sweeping the conductance domain from small to large $G$ values, $\Gamma_{\mathrm{VNA}}(G)$ first decreases to reach zero reflectance at the matching point where $G=G_{\text {match }}$, then increases back for $G>G_{\text {match. }}$ The fit together with the measured scatter plot is shown in Fig. 2(c).

\section{NOISE-MEASUREMENT CALIBRATION}

The current noise produced by a QD can be modeled by a noise voltage source $V_{\text {noise }}$ as shown in Figs. 1(d) and 1(e). The power spectral density of the voltage source $S_{V}$ is related to current spectral density $S_{I}$ by $S_{V}=R^{2} S_{I}$. 
The noise voltage needs to be transmitted through the $L C$ circuit to be fed into the $Z_{0}$-transmission line. The voltage $V$ that appears at the interface is proportional to $V_{\text {noise }}$ but contains, in addition, a frequency-dependent transmission function $t_{V}(f) \equiv V / V_{\text {noise }}$, which can readily be derived from the $L C$-circuit parameters:

$$
t_{V}(\Omega) \approx \frac{Z_{0} G}{1+i \Omega\left(\frac{Z_{0}}{Z_{c}}+\frac{R_{\text {loss }}}{Z_{c}}+\frac{Z_{c}}{R}\right)-\Omega^{2}},
$$

where $\Omega=\omega / \omega_{0}=2 \pi f \sqrt{L C}$, and $Z_{c}=\sqrt{L / C}$ is the characteristic impedance, as introduced before. In this equation, the factor of $i \Omega$ shows that the total $Q$ factor has three terms, which can be grouped in an internal-loss part $1 / Q_{\text {int }}$ and an external-loss part $1 / Q_{\text {ext }}$. The two parts are $1 / Q_{\text {int }}=R_{\text {loss }} / Z_{c}$ and $1 / Q_{\text {ext }}=Z_{0} / Z_{c}+Z_{c} / R$, respectively. At optimal impedance matching in the lossless picture-i.e., if the condition $Z_{c}=\sqrt{R Z_{0}}$ is met-the external $Q$ factor is minimal and is given by $\sqrt{R / Z_{0}} / 2$, which yields values of around 10-100.

The transmitted voltage passes several elements, a directional coupler, a circulator, and amplifiers at low and room temperature before being measured with the PSA. The overall gain along the chain is captured by $g$. It is determined using the method presented in Ref. [14] to be $g=94.6 \mathrm{~dB}$. In addition to the noise generated by the device, the amplifier also adds further current-independent noise. We split the total measured noise power, $\langle\delta P\rangle$, into two contributions:

$$
\langle\delta P\rangle=\frac{S_{I} R^{2}}{Z_{0}} g \int_{\mathrm{BW}}\left|t_{V}(f)\right|^{2} d f+\left\langle\delta P_{0}\right\rangle,
$$

where the first term describes the shot noise originating in the QD [4] and the second term, $\left\langle\delta P_{0}\right\rangle$, contains the rest (the thermal noise arising from the device and the amplifier noise background).

\section{EXPERIMENT}

By means of gate- and bias-voltage sweeps, we measure the dc current and draw an initial charge-stability diagram. Then we switch to MW-reflectance measurements, where we restrict ourselves to a gate span covering a few Coulomb charge states. In this measurement mode, we simultaneously acquire the current $I$ and the reflectance $\left|\Gamma_{\mathrm{VNA}}\right|^{2}$ as functions of $V_{S D}$ and $V_{G}$. The VNA is set to the working frequency $f_{m}=3.23 \mathrm{GHz}$ with an output power of $-25 \mathrm{dBm}$, attenuated by about $87 \mathrm{~dB}$ before reaching the device. We deduce the parameters of the $L C$ impedance-matching circuit from a comparison of the two measured maps $d I / d V_{S D}\left(V_{G}, V_{S D}\right)$ [Fig. 3(a)] and $\left|\Gamma_{\mathrm{VNA}}\left(V_{G}, V_{S D}\right)\right|^{2}$ [Fig. 3(b)], as described in the previous section. In practice, the conductance domain of the resulting $\left|\Gamma_{\mathrm{VNA}}\left(d I / d V_{S D}, f_{m}\right)\right|^{2}$ scatter plot does not include
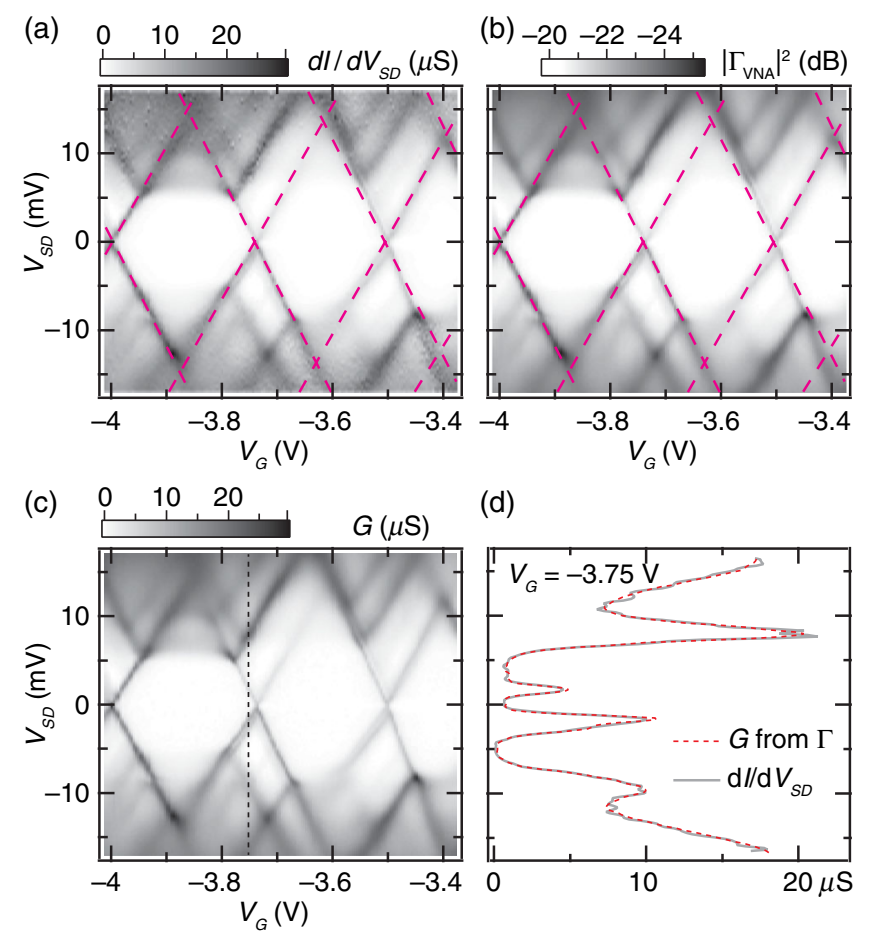

(d)

FIG. 3. (a) Derivative of the dc current $\left(d I / d V_{S D}\right)$ as a function of the gate and source-drain voltages. The contour of the Coulomb diamonds is highlighted by the dashed line. (b) Reflectance measured at $f=3.23 \mathrm{GHz}$, with the same contour as in (a). (c) Differential conductance $G$ deduced from the reflectance $\left|\Gamma_{\mathrm{VNA}}\right|^{2}\left(f=3.23 \mathrm{GHz}, V_{G}, V_{S D}\right)$ by using the $L C$ matchingcircuit parameters extracted in the fitting. (d) Cuts in the (a),(c) maps, showing an excellent overlap of low- and high-frequency differential-conductance values.

values close to $G_{\text {match }}$; therefore, we enrich the plot with a close-to-match point, $\left|\Gamma_{\mathrm{VNA}}^{\text {near match }}\left(f_{m}\right)\right|^{2}$, from the separately obtained near-match curve in Fig. 2(b).

From complementary measurements, we estimate an upper bound of $R_{\text {loss }} \approx 1 \Omega$ (see the Supplemental Material [15]). Since $R_{\text {loss }} \ll Z_{0}$, the matching circuit can be modeled as a lossless $L C$ circuit for practical purposes. Thus, we fix $R_{\text {loss }}=0$ and fit the curve $\left.\left|\Gamma_{\mathrm{VNA}}\right|^{2}\left(G, f=f_{m}\right)\right]$ Fig. 2(c)], which yields $L=37 \mathrm{nH}, C=63 \mathrm{fF}$. We further deduce the resonance frequency $f_{0}=3.28 \mathrm{GHz}$ and the characteristic impedance $Z_{c}=766 \Omega$; the resulting match conductance is the minimum of the calculated curve $\left|\Gamma_{\mathrm{VNA}}\right|^{2}\left(G, f=f_{0}\right)$ : $G_{\text {match }}=84 \mu \mathrm{S}$. For comparison, refitting with $R_{\text {loss }}=1 \Omega$, the calculated $L, C$, and $G_{\text {match }}$ vary by less than $1 \%$. Furthermore, for $R_{\text {loss }} \leq 1 \Omega$, we obtain $Q_{\text {ext }} \leq 15$ and $Q_{\text {in }} \geq 766$, yielding an empirical bandwidth $f_{0} / Q \gtrsim$ $220 \mathrm{MHz}$.

Consequently, having fixed the parameters, we then calculate a full map of the real part of the device admittance, i.e., $G\left(V_{G}, V_{S D}\right)$ [Fig. 3(c)]. A comparison of cuts [Fig. 3(d)] demonstrates very good agreement with $d I / d V_{S D}$, confirming the validity of the extracted parameters, at least at the frequency at which the 

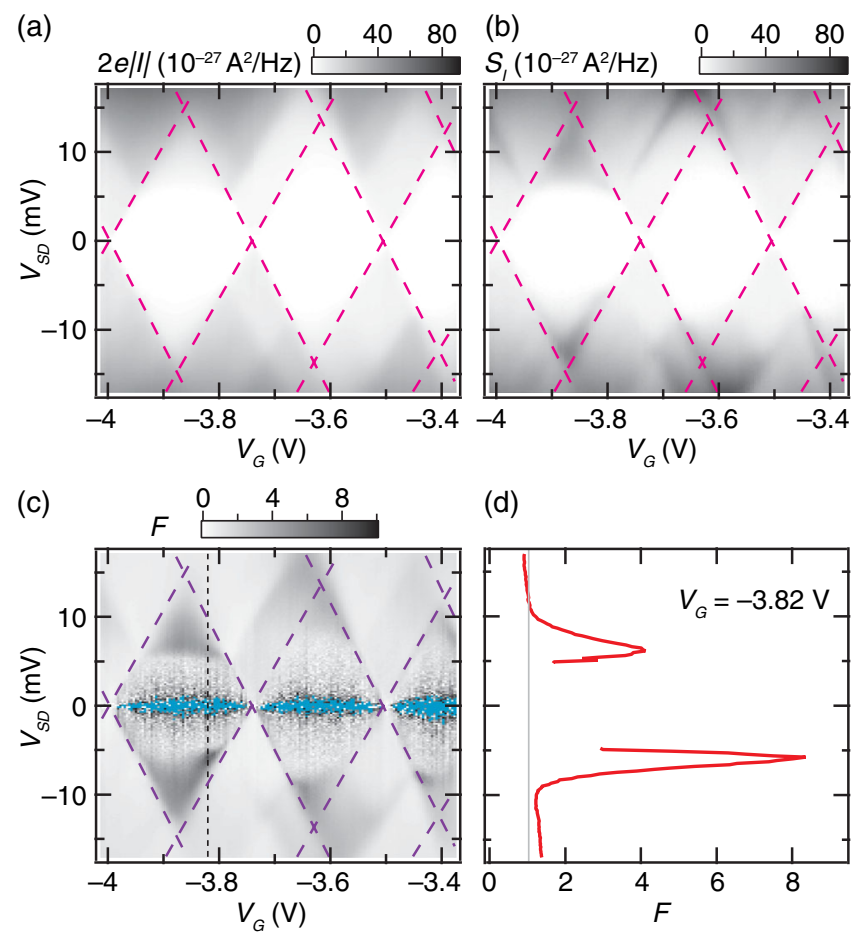

(d)

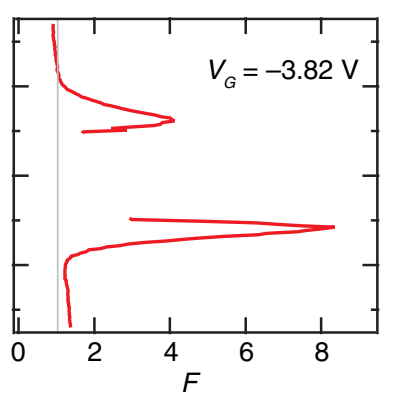

FIG. 4. (a) Shot-noise spectral density $S_{I}$ as a function of $V_{G}$ and $V_{S D}$ obtained from the measured power spectral density $\langle\delta P\rangle$ at the power spectrum analyzer using Eq. (6). (b) Schottky noise $2 e|I|$ calculated from the dc measured current $I$, and (c) Fano factor $F=S_{I} /|2 e I|$. The Coulomb-diamond contours (the dashed lines) are copied from the conductance plot in Fig. 3(a). Instead of the $F=1$ value proper for elastic cotunneling, the low-bias cyan regions indicate erroneously calculated values emerging from the division of a "noisy" number by a very small one. These regions need to be discarded. A cut at the black dashed vertical line is depicted in (d). The observed highest Fano factor is $F \simeq 8$.

reflectance map is acquired. The advantage of the reflectance measurement is that $G$ can be measured much faster and with less noise once the circuit parameters are known.

In the noise part of the experiment, we realize simultaneous current and noise measurements over the same fewCoulomb-diamond span as before. Each retained noise power value $\langle\delta P\rangle$ arises from averaging 500 identical input measurements in a bandwidth $\mathrm{BW}=50 \mathrm{MHz}$, and it is produced approximately every $800 \mathrm{~ms}$. Figure 4(b) shows the current noise $S_{I}$ measured in the same gate range after applying Eq. (6) for calibration. To make a comparison to the Schottky noise, we show in Fig. 4(a) a calculated plot of $2 e|I|$, where $I$ represents the measured dc current. The Fano factor, $F \equiv S_{I} /|2 e I|$, is shown in Fig. 4(c). We observe values largely exceeding 1 within the Coulomb blockade, where sequential tunneling of single electrons is forbidden. It is seen in Fig. 4(d) that the Fano factor peaks at $V_{S D} \approx \pm 5 \mathrm{mV}$, where inelastic cotunneling sets in. Such a cotunneling event can excite the QD, enabling the sequential transfer of single electrons through the QD until the QD decays back to the ground state [16-19].
The respective train of charge bunches leads to a large Fano factor, which here reaches values as large as 8 . To date, only super-Poissonian noise with $F \leq 3$ has been reported in QDs [20,21]. At lower voltage bias, solely elastic cotunneling is present and the Fano factor should be 1 [17,21], this being the case in those regions of the second Coulomb diamond closer to the inelastic cotunneling onset; at an even lower bias, the calculation of $F$ produces divergent values here due to the division of noisy numbers by the smallest current numbers, such that $F>10$ erroneously in the cyan domains of Fig. 4(c).

\section{DISCUSSION AND CONCLUSION}

In this work, we successfully demonstrate the realization and application of a QD-impedance-matching on-chip superconducting coil working at $3.25 \mathrm{GHz}$. We deduce the $L C$-circuit parameters by using the dependence of the reflectance $|\Gamma|^{2}$ on the device resistance $R$ at a fixed frequency. The alternative approach, where one fits the frequency dependence of $|\Gamma|^{2}$ to the expected functional form for a fixed value $R$-typically at $G=1 / R=0$ deep in the Coulomb-blockade regime-turns out to be less reliable due to the large bandwidth offered by the $L C$ circuit. Consequently, the circuit is affected by a frequencydependent background $b(f)$ due to, for example, spurious standing waves in parts of the MW lines. Compared to transmission-line resonators used as impedance-matching circuits, the figure of merit $g_{\mathrm{SNR}}=\mathrm{SNR} / \mathrm{SNR}_{0}$, where $\mathrm{SNR}_{0}$ is the signal-to-noise ratio in the absence of an impedance-matching circuit [4], is shown to be the same for, e.g., a stub tuner and an $L C$ circuit if one acquires the noise over the full bandwidths given by the FWHMs of the respective transfer functions $t_{V}(f)$ [14]. The advantage of the $L C$ circuit lies in its larger bandwidth, approximately $100 \mathrm{MHz}$, compared to a transmission line resonator. This bandwidth increase is important in applications where short interaction times are essential, i.e., for fast readout and qubit manipulation. The disadvantage is that one has to account for spurious resonances in the external system leading to a frequency-dependent background. This alteration can be a problem if highly accurate pulses need to be transmitted for qubit manipulation. Residual and uncontrolled phase shifts may make it difficult to achieve high gate fidelities. For noise measurement, we show that it is a powerful circuit.

\section{ACKNOWLEDGMENTS}

We acknowledge financial support from the ERC project QUEST and the Swiss National Science Foundation (SNF) through various grants, including NCCR-QSIT. We are grateful to Fabien Portier for the insightful discussions regarding the design and fabrication of microwave resonators. We also thank Lukas Gubser for the useful discussions. 
[1] V. E. Manucharyan, J. Koch, L. I. Glazman, and M. H. Devoret, Fluxonium: Single Cooper pair circuit free of charge offsets, Science 326, 113 (2009).

[2] Gabriel Puebla-Hellmann and Andreas Wallraff, Realization of gigahertz-frequency impedance-matching circuits for nano-scale devices, Appl. Phys. Lett. 101, 053108 (2012).

[3] V. Ranjan, G. Puebla-Hellmann, M. Jung, T. Hasler, A. Nunnenkamp, M. Muoth, C. Hierold, A. Wallraff, and C. Schönenberger, Clean carbon nanotubes coupled to superconducting impedance-matching circuits, Nat. Commun. 6, 7165 (2015).

[4] T. Hasler, M. Jung, V. Ranjan, G. Puebla-Hellmann, A. Wallraff, and C. Schönenberger, Shot Noise of a Quantum Dot Measured with Gigahertz Impedance Matching, Phys. Rev. Applied 4, 054002 (2015).

[5] R. Schoelkopf, P. Wahlgren, A. Kozhevnikov, P. Delsing, and D. Prober, The radio-frequency single-electron transistor (RF-SET): A fast and ultrasensitive electrometer, Science 280, 1238 (1998).

[6] Ji Chen and Juin J. Liou, On-chip spiral inductors for RF applications: An overview, J. Semicond. Technol. Sci. 4, 149 (2004).

[7] Chik Patrick Yue, Ph.D. thesis, Stanford University, 1998, p. 36.

[8] W. W. Xue, B. Davis, Feng Pan, J. Stettenheim, T. J. Gilheart, A. J. Rimberg, and Z. Ji, On-chip matching networks for radio-frequency single-electron transistors, Appl. Phys. Lett. 91, 093511 (2007).

[9] Kin Chung Fong and K. C. Schwab, Ultrasensitive and Wide-Bandwidth Thermal Measurements of Graphene at Low Temperatures, Phys. Rev. X 2, 031006 (2012).

[10] W. W. Xue, Ph.D. thesis, Dartmouth College, 2010, p. 79.

[11] Carles Altimiras, Olivier Parlavecchio, Philippe Joyez, Denis Vion, Patrice Roche, Daniel Esteve, and Fabien Portier, Tunable microwave impedance matching to a high impedance source using a Josephson metamaterial, Appl. Phys. Lett. 103, 212601 (2013).

[12] Anna Stockklauser, Pasquale Scarlino, Jonne V. Koski, Simone Gasparinetti, Christian Kraglund Andersen, Christian Reichl, Werner Wegscheider, Thomas Ihn, Klaus Ensslin, and Andreas Wallraff, Strong Coupling Cavity QED with GateDefined Double Quantum Dots Enabled by a High Impedance Resonator, Phys. Rev. X 7, 011030 (2017).

[13] J. J. Viennot, J. Palomo, and T. Kontos, Stamping single wall nanotubes for circuit quantum electrodynamics, Appl. Phys. Lett. 104, 113108 (2014).

[14] See the Supplemental Material of Ref. [4].

[15] See Supplemental Material at http://link.aps.org/ supplemental/10.1103/PhysRevApplied.8.054006 for the loss characterization and the fitting method.

[16] M. R. Wegewijs and Yu. V. Nazarov, Inelastic co-tunneling through an excited state of a quantum dot, arXiv:cond-mat/ 0103579.

[17] Eugene V. Sukhorukov, Guido Burkard, and Daniel Loss, Noise of a quantum dot system in the cotunneling regime, Phys. Rev. B 63, 125315 (2001).

[18] I. Weymann, J. Barnaś, and S. Krompiewski, Transport through single-wall metallic carbon nanotubes in the cotunneling regime, Phys. Rev. B 78, 035422 (2008).

[19] S. Gustavsson, M. Studer, R. Leturcq, T. Ihn, K. Ensslin, D. C. Driscoll, and A. C. Gossard, Detecting single-electron tunneling involving virtual processes in real time, Phys. Rev. B 78, 155309 (2008).

[20] E. Onac, F. Balestro, B. Trauzettel, C. F. J. Lodewijk, and L. P. Kouwenhoven, Shot-Noise Detection in a Carbon Nanotube Quantum Dot, Phys. Rev. Lett. 96, 026803 (2006).

[21] Yuma Okazaki, Satoshi Sasaki, and Koji Muraki, Shot noise spectroscopy on a semiconductor quantum dot in the elastic and inelastic cotunneling regimes, Phys. Rev. B 87, 041302(R) (2013). 